\title{
Corrigendum: Merging of metal nanoparticles driven by selective wettability of silver nanostructures
}

Michael Grouchko, Polina Roitman, Xi Zhu, Inna Popov, Alexander Kamyshny, Haibin Su \& Shlomo Magdassi

Nature Communications 5:2994 doi: 10.1038/ncomms3994 (2014); Published 6 Jan 2014; Updated 28 Jan 2014

The funding for this Article was not acknowledged. The Acknowledgements should have read:

This work is supported by the Singapore National Research Foundation under the CREATE program: Nanomaterials for Energy and Water Management, and by the Israel National Nanotechnology Initiative. 